\title{
Sensitivity Analysis of a Simulation Model for Managing Contact Centers with Impatient Customers
}

\author{
Chia-Hung Wang ${ }^{1,2, *}$ and Bin $\mathrm{Zhu}^{1}$ \\ ${ }^{1}$ College of Information Science and Engineering, Fujian University of Technology, Fuzhou City, Fujian Province 350118, China \\ ${ }^{2}$ Fujian Provincial Key Laboratory of Big Data Mining and Applications, Fuzhou City, Fujian Province 350118, China \\ ${ }^{*}$ Corresponding author
}

\begin{abstract}
Having worked as the primary contact points among customers and companies in the last decades, contact centers become a great investment for many organizations. This paper aims to study a queuing system with abandonments for managing the modern contact centers, where the impatient customers would probably abandon their calls if their patience expires before they reach a service agent. We develop an Arena simulation model to determine the system performance of interest. To figure out some useful managerial insights, we conduct a sensitivity analysis of the proposed system through a series of numerical experiments. The contribution of this study is to help managers achieve a better understanding of the optimal design and operation at the real-world contact centers.
\end{abstract}

Keywords-arena simulation; queueing system; contact center; balking; reneging

\section{INTRODUCTION}

With the increasing development of e-business, contact centers have become important connections between enterprises and customers. The contact center industry is vast and rapidly expanding in terms of both workforce and economic scope. According to the report of [1] in Year 2015, it was estimated that China's contact/call center market reached 110.5 billion yuan, and the total staffing number at China's call centers reached 961.1 thousand. Modern contact centers are challenged with multitude types of calls, which come in over different communication channels (telephone, Internet, fax, e-mail, chat, mobile devices, etc.); meanwhile, agents need to have the skill to handle one or more types of calls.

A key challenge in designing and managing contact centers with impatient customers is to achieve a desired balance between operational efficiency and service quality. The service level of the real-world contact centers is generally targeted as $80 / 20$, which represents that $80 \%$ of customers can receive service in 20 seconds. A natural consequence of customers' dissatisfaction with the system performance is that customers may lose their patience and abandon the queue. One of the managerial approaches to achieve the service level is to provide the delay time information to the customers in order to influence their impatience behaviors (such as balking and reneging) and the operational efficiency of contact centers [2]. Therefore, it is important for managers of contact centers to have an efficient quantitative approach to evaluate the system performance [3].
Simulation methods have always played an important role in the design and management of contact centers in the last decades, e.g., [4], [5] and references therein. In a contact center system, a virtual queue line occurs when there is no service agent available to handle a customer's call. However, a few contact center characteristics may make it difficult to apply the analytical methods (such as Queue Theory) for its modeling, including: generic distribution for the handling time, time-varying arrival rates, temporary overflows and abandonment [3]. At this point, the simulation models are effective in measuring the performance of a given (real-world) contact center. Besides, the contact centers can use the simulation tools to test whether some changes can prove or not to be able to improve the system before its implementation.

In this paper, we propose a simulation model of contact center to study the influence of customer's impatience behaviors on the system performance. Here, the customers' impatience behaviors include direct balking and reneging in the waiting queue. The aim of this study is to perform a sensitivity analysis of the proposed simulation model for managing the contact centers with impatient customers. The properties to be figured out in our experimental results could contribute managerial insights for optimizing the parameter settings of the proposed simulation model. In the face of potential real-world scenarios, the managers and analysts of contact centers may use the proposed simulation model effectively to design their systems, manage the operation and plan ahead.

The remainder of this paper is organized as follows: In Section 2, we review some existed works on the application of simulation methods to manage the contact centers. In Section 3 , a simulation model is developed via the Arena simulation software for studying the proposed queueing system with impatient customers. We conduct a series of sensitivity analysis in Section 4 to demonstrate the effect of interested model parameters on the system performance. Finally, our concluding remarks are summarized in Section 5.

\section{LITERATURE REVIEW}

There are plenty of existed research works where simulation methods were proposed as the most appropriate option for accurate performance evaluations and decision makings for managing the (real-world) contact centers. Through the use of simulation, we can translate the gross data of a contact center into several important performance 
measures, e.g., handling information on the service levels, call abandonment, the number of agents, and costs, etc. Mehrotra and Fama [6] provided an overview of call/contact center simulation models, which highlighted typical inputs and data sources, modeling challenges, and key model outputs. Wallace and Saltzman [7] used two distinct simulation programming methods (the $\mathrm{C}$ language and the Arena software package) to describe the modeling of a skill-based routing call center, and provided a comparison of the pros and cons of using each simulation programming approach.

Sencer and Ozel [8] showed that the combination of the advantages of simulation with a flexible and user-friendly decision support system environment provided more effective and efficient workforce planning and performance reporting in call centers. Wang and Hung [9] applied Arena simulation software to study the optimal design and staffing problems in call centers. Atlason et al. [10] present a simulation-based analytic center cutting-plane method to study the staffing problem in an inbound call center, while maintaining an acceptable level of service in multiple time periods.

In the heavy traffic of a certain contact center, even a small fraction of busy-signals in queues or customers' abandonment could have a dramatic effect on performance, and hence must be taken into account. The operational service level of a given contact center is typically quantified in terms of some congestion or performance measures, and Koole and Mandelbaum [11] suggest a managerial focus on the customers' abandonment rate and waiting time. For studying queueing models with abandonments, Zeltyn and Mandelbaum [12] applied the $M / M / n+G$ queueing model characterized by Poisson arrivals, exponential service times, finite service agents and generally distributed patience times of customers in the call center environment. For staffing contact/call centers with impatient customers, Zhang et al. [13] presented refinements to many-server asymptotics and the square-root staffing principle for a Markovian queueing model with impatient customers.

\section{An AREna Simulation Model}

A simulation model is developed in our study via the Arena simulation software to evaluate the system performance of the contact center with impatient customers. In the proposed model of a contact center, the customers are callers, and the queues consist of callers that await service by a system resource. As shown in Figure 1, our Arena simulation model is a collection of data modules, logic modules and process modules, which contain all of the required model parameters, logic, and animation necessary to describe its specific portion of the studied system.

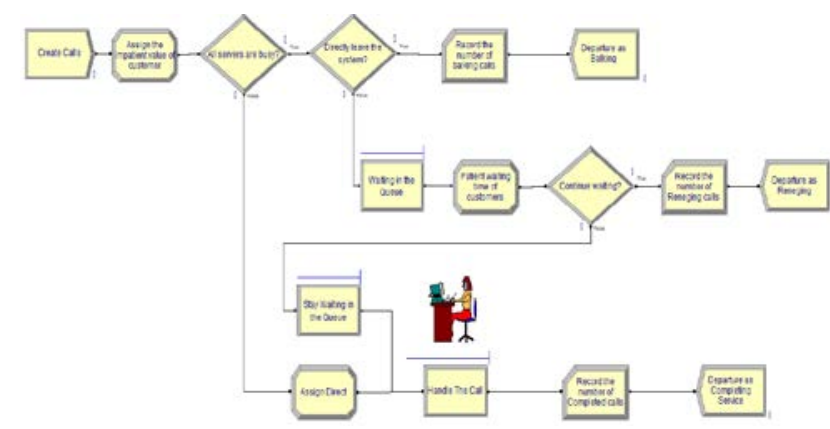

FIGURE I.AN ARENA SIMULATION MODEL FOR A CONTACT CENTER WITH IMPATIENT CUSTOMERS.

In the studied simulation model, we assume that customers would probably abandon their calls if their patience expires before they reach an agent (server) at the contact center. We assign reasonable parameters to their patience-time distributions, and assume that the patience-time distribution has an atom at the origin. In this system, there are two types of abandonment probabilities, i.e., the balking probability and the reneging probability. We define the balking probability as the fraction of customers who refuse to wait at all. We define the reneging probability as the fraction of waiting customers who explicitly declare that the service offered is not worth its waits.

When a customer's call arrives at the system, the customer obtains the service directly if the system has (at least) one idle service agent; otherwise, the customer directly leave the system (Balking) with probability $\alpha$, where $0 \leq \alpha \leq 1$. For those customers who don't choose to leave directly, he/she will enter the waiting queue with the probability $1-\alpha$. When the waiting time of a customer waiting in the queue is greater than his/her patience value, the customer will choose to leave system (Reneging) with the probability $\beta$, or choose to wait in the queue until receiving his/her service with probability $1-\beta$, where $0 \leq \beta \leq 1$. We assume the customers' patience value follows the exponential distribution with the parameter $\theta$ and is independent.

In our model, we define the ratio of completing-service calls as the proportion of the number of served calls to the total number of calls generating in a specified (simulation) time. We also define the abandonment rate as the ratio of the number of abandoned calls to the total number of customers' calls. Customers' calls are served on a first-come-first-served basis.

For the proposed Arena simulation model, we will determine the average waiting time, the ratio of completingservice calls and the abandonment rate through a series of simulation experiments in Section 4. It will demonstrate the performance effect of the interested model parameters (the balking probability $\alpha$, the reneging probability $\beta$, and the average customers' patience value $\theta$ ) on the studied system.

\section{NUMERICAL RESULTS}

In this section, we conduct the sensitivity analysis to study the managerial effect of the interested model parameters on the system performance of the proposed Arena simulation model. Our numerical experiments are run through Arena 
simulation software version 14.00 on the PC platform with Intel Core i5-2520M (2.5 GHz) and 8 GB RAM. In a series of computer simulation experiments, our Arena model parameters are set as follows: the average number of customers' calls is 48 per minute, the number of agents is 45 , the average service time per agent is 1 minute, the balking probability $\alpha$ varies in the interval $[0,1]$, the reneging probability $\beta$ varies in the interval $[0,1]$, and the average customers' patience value $\theta$ varies in the interval [0.1,3.9].

In the following simulation experiments, we observe the effect on the interested system performance by varying two abandonment rates (balking probability $\alpha$, reneging probability $\beta$ ) and the average customers' patience (time) value $\theta$, respectively. Our computer simulation was carried out with long enough time during each simulation experiment. Each simulation experiment was repeated 10 times independently, and those numerical results depicted in Figures 2-7 are obtained by averaging those values of 10 experimental data.

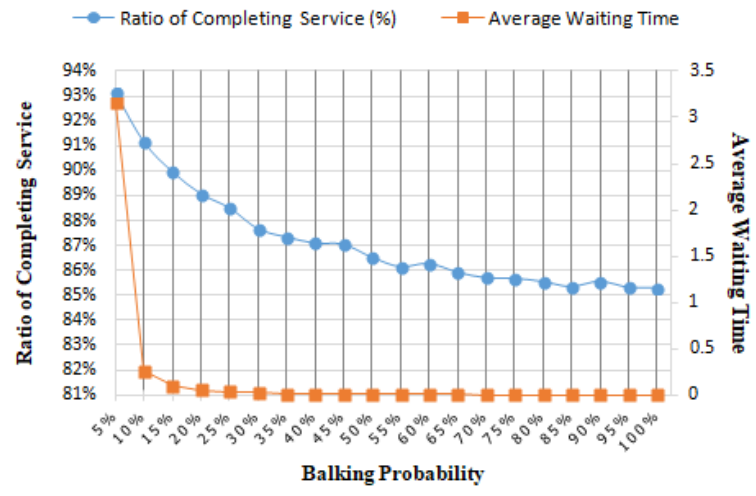

FIGURE II. TWO PERFORMANCE INDICATORS VERSUS THE BALKING PROBABILITY.

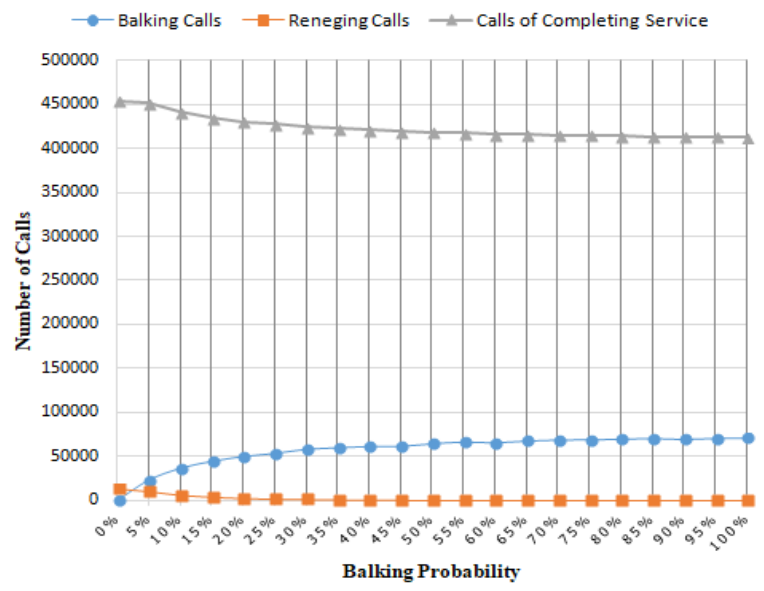

FIGURE III

THE NUMBER OF ABANDONMENT CALLS VERSUS THE BALKING PROBABILITY.

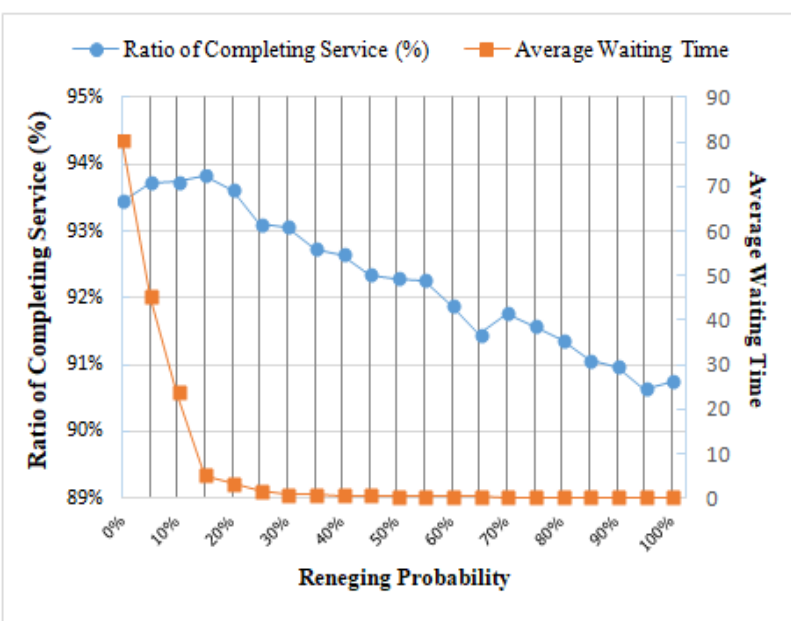

FIGURE IV. TWO PERFORMANCE INDICATORS VERSUS THE RENEGING PROBABILITY

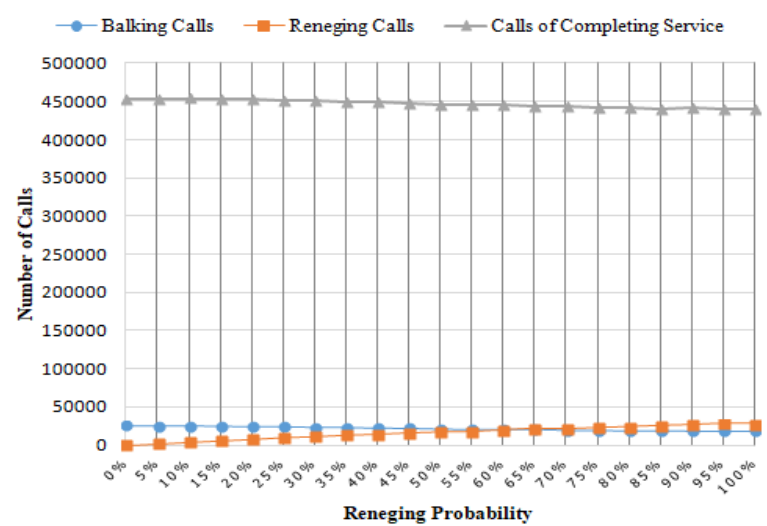

FIGURE V. THE NUMBER OF ABANDONMENT CALLS VERSUS THE RENEGING PROBABILITY

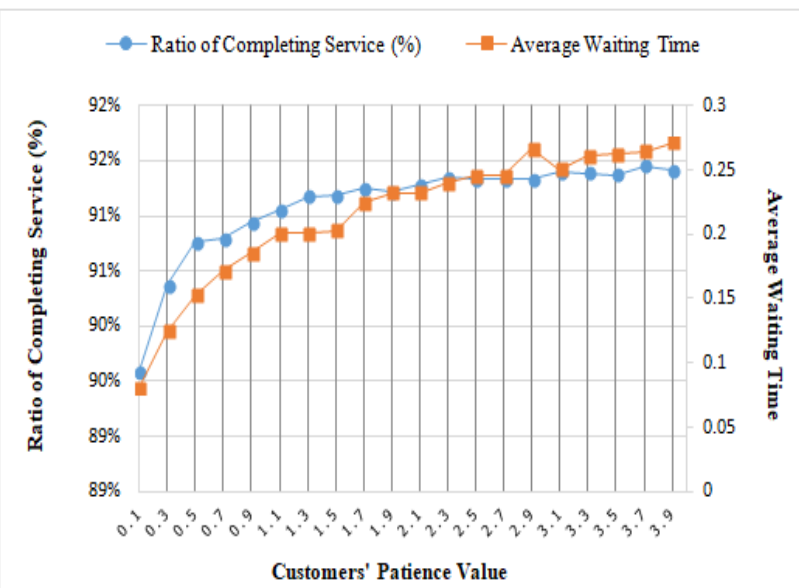

FIGURE VI. TWO PERFORMANCE INDICATORS VERSUS THE AVERAGE PATIENCE VALUE OF CUSTOMERS 


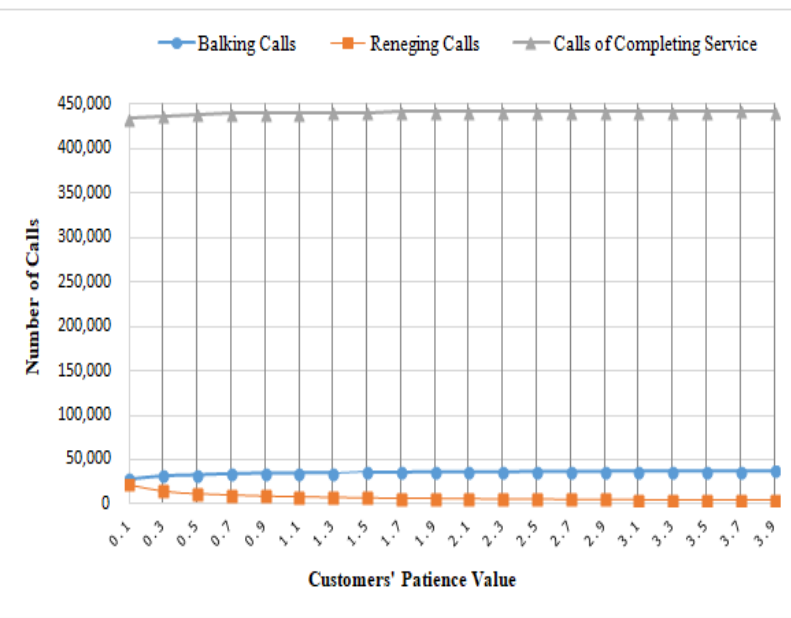

FIGURE VII. THE NUMBER OF ABANDONMENT CALLS VERSUS THE AVERAGE PATIENCE VALUE OF CUSTOMERS

\section{A. The Effect of the Balking Probability}

First, we observe the effect of varying the balking probability $\alpha$ on the system performance given that the reneging probability $\beta=0.25$ and the average customers' patience value $\theta=2$ are fixed. Figure 2 demonstrates that it decreases the ratio of completing-service calls and the average waiting time if we increase the balking probability $\alpha$. In Figure 3 , it illustrates that the abandonment rate will be increased as increasing the balking probability.

\section{B. The Effect of the Reneging Probability}

Next, we observe the influence of changing the reneging probability $\beta$ on the system performance given that the balking probability $\alpha=0.05$ and the average customers' patience value $\theta=2$ are fixed. In Figure 4, it shows that if we increase the reneging probability, both the ratio of completing-service calls and the average waiting time will be decreased. Figure 5 illustrates that it increases the total number of reneging calls and decreases the total number of balking calls when we increase the balking probability.

\section{The Effect of the Average Customers' Patience Value}

Finally, we observe the effect of the average customers' patience value $\theta$ on the system performance given $\alpha=0.1$ and $\beta=0.25$. In Figure 6 , we observe that both the ratio of completing-service calls and the average waiting time are increased if we increase the average patience value $\theta$. In Figure 7 , it can be found that the abandonment rate will be decreased as increasing the average patience value $\theta$.

From the above sensitivity analysis in Figures 2-7, it demonstrates that there are several monotone trends in the system performance of the proposed simulation model when changing the balking probability $\alpha$, the reneging probability $\beta$, and the average customers' patience value $\theta$, respectively.

\section{CONCLUSIONS}

This paper studies the impact of customers' impatient behaviors (in terms of balking and reneging) on the system performance of a contact center. We developed an Arena simulation model to evaluate the performance indicators in the proposed system, such as the average waiting time, the ratio of completing-service calls and the abandonment rate of customers' calls. To investigate the sensitivity analysis of the system performance, we have conducted a series of numerical experiments via varying different model parameters of interest. From our experimental data, we find that there are several significant trends in the change of customers' average waiting time and the average number of abandonment calls.

According to the sensitivity analysis presented in this study, some managerial insights would be contributed into the optimal design and operation when managing the contact centers with impatient customers. Our findings could also be helpful in balancing the trade-off between the operational costs and the quality of service offered to customers. In the practical applications, the contact center managers could use the proposed simulation model to track the interested performance indicators and then react effectively when those indicators reach the unacceptable levels.

\section{ACKNOWLEDGMENT}

This research was supported in part by Fujian Provincial Department of Science and Technology, China, under Grant No. 2016J01330, and the Education Department of Fujian Province, China, under Grant No. JAT160337.

\section{REFERENCES}

[1] Zero Power Intelligence Group, Research and Consultation Report of Panorama Survey and Investment Strategy on China's Call Center Industry in Year 2015-2020, Beijing, China, 2015. Available at http://www.chinairn.com

[2] Z. Akşin, B. Ata, S.M. Emadi, and C.-L. Su, "Impact of delay announcements in call centers: an empirical approach,” Oper. Res., vol. 65, no. 1, pp. 242-265, 2017.

[3] M.A.C. Bouzada, "Dimensioning a call center: simulation or queue theory?,” J. Oper. Supply Chain Manag., vol. 2, no. 2, pp 34-46, 2009.

[4] P. Dileepan and L.P. Ettkin, "Not just for large companies: benefits of simulation modeling for a small telephone call center,” Prod. Inventory Manage. J., vol. 1, pp. 54-63, 2010.

[5] A.D. Leva, E. Sulis, and M. Vinai, "Business process analysis and simulation: the contact center of a public health and social information office,” Intel. Info. Manage., vol. 5, pp. 189-205, 2017.

[6] V. Mehrotra and J. Fama, "Call center simulation modeling: methods, challenges and opportunities,” Proc. 2003 Winter Simul. Conf., pp. 135143, 2003.

[7] R.B. Wallace and R.M. Saltzman, "Comparing skill-based routing call center simulations using C programming and Arena models”, Proc. 2005 Winter Simul. Conf., pp. 2636-2644, 2005.

[8] A. Sencer and B.B. Ozel, "A simulation-based decision support system for workforce management in call centers,” Simul.- Trans. Soc. M. S., vol. 89, no. 4, pp. 481-497, 2013.

[9] C.-H. Wang and M.-H. Hung, "Simulation model and optimal design for call center staffing problems,” Adv. Intell. Syst., vol. 536, pp. 103-110, 2016.

[10] J. Atlason, M.A. Epelman, and S.G. Henderson, “Optimizing call center staffing using simulation and analytic center cutting-plane methods," Manage. Sci., vol. 54, no. 2, pp. 295-309, 2008.

[11] G. Koole and A. Mandelbaum, "Queueing models of call centers: an introduction,” Ann. Oper. Res., vol. 113, pp. 41-59, 2002. 
[12] S. Zeltyn and A. Mandelbaum, "Call centers with impatient customers: many-server asymptotics of the $\mathrm{M} / \mathrm{M} / \mathrm{n}+\mathrm{G}$ queue,” Queueing Syst., vol. 51, pp. 361-402, 2005.

[13] B. Zhang, J.S.H. van Leeuwaarden, and B. Zwart, "Staffing call centers with impatient customers: refinements to many-server asymptotics," Oper. Res., vol. 60, no. 2, pp. 461-474, 2012. 